Mini-review

\title{
The impact of Mesenchymal Stem Cells and their secretome as a treatment for gliomas
}

\author{
E.D. Gomes ${ }^{\text {a, b, } 1 \text {, J. Vieira de Castro }}{ }^{\text {a, b, } 1 \text {, B.M. Costa }}{ }^{\text {a, b, } 2}$, A.J. Salgado ${ }^{\text {a, b, }}$ *, 2 \\ ${ }^{a}$ Life and Health Sciences Research Institute (ICVS), School of Medicine, University of Minho, Campus de Gualtar, 4710-057, Braga, Portugal \\ ${ }^{\mathrm{b}}$ ICVS/3B's - PT Government Associate Laboratory, Braga/Guimarães, Portugal
}

\section{A R T I C L E I N F O}

\section{Article history:}

Received 13 February 2018

Accepted 16 July 2018

Available online xxx

\section{Keywords:}

Mesenchymal stem cells

Secretome

Cancer therapy

Glioblastoma

\begin{abstract}
A B S T R A C T
In recent years, we have witnessed a significant increase in the amount of studies using Mesenchymal Stem Cells (MSCs) for cancer therapy, mostly as vectors for drug or gene delivery strategies. This is because of their intrinsic capacity of homing into tumor niches. However, the interactions between MSCs themselves and tumor cells is not fully understood, with contradictory results frequently being observed regarding their effects on cancer cell invasion and proliferation. This poses an important question of safety in respect to the application of these cells. The source of the MSC population used, as well as the type of cancer cells under study might strongly influence this interaction. Moreover, differences in isolation protocols, culture media compositions, time of culture and conditioned media collection, or even timing and mode of MSCs administration to in vivo models of cancer may also affect the interaction MSC-tumor cells. In this review, we drive our focus into malignant brain tumors, particularly gliomas, one of the deadliest forms of cancer. Moreover, we look with some detail into different studies using MSCs as a treatment for brain tumors and compare them, highlighting the main deviations and similarities among them.
\end{abstract}

๑ 2018 Elsevier B.V. and Société Française de Biochimie et Biologie Moléculaire (SFBBM). All rights reserved.

\section{Introduction}

Malignant gliomas are the most common primary brain tumor (representing $80 \%$ of all malignant brain tumors) and one of the deadliest forms of cancer [1]. It is believed that they have a glial origin or derive from stem/progenitor cells that undergo malignant transformation [2]. Glioma mainly develops in the brain, although it can also develop in other parts of the CNS. Glioblastoma (GBM) is the most common and aggressive subtype of glioma. Current treatment techniques are based on tumor resection followed by sessions of radio and/or chemotherapy. However, despite all the efforts developed in the last decades, patients' prognosis has only increased a few months [3]. One of the reasons for the high mortality rates registered and for the difficulties found in treating GBM

\footnotetext{
* Corresponding author. António Salgado Life and Health Sciences Research Institute - ICVS School of Medicine University of Minho, Campus de Gualtar, 4710057, Braga, Portugal.

E-mail address: asalgado@med.uminho.pt (A.J. Salgado).

1 Equally contributing.

2 Shared Senior authorship.
}

is the tumor's infiltrative capacity. The tumor cells possess a high proliferative and invasive profile, which makes complete surgical resection virtually impossible and increases the probability of tumor recurrence. Recent evidences suggest that remarkable tumor heterogeneity, and poor response to current therapies are also partly due to the existence of cancer stem cells (CSCs). Similar to other stem cells, CSCs have a self-renewal capacity and can differentiate into different cell phenotypes, originating the main cell populations forming the tumor [4].

The development of new therapeutic options, capable of targeting not only the "bulk" tumor cells but also CSCs, remains therefore an important need.

The application of Mesenchymal Stem Cells (MSCs) in different therapeutic approaches is becoming common in recent years. The identification of these cells is credited to the work of Friedenstein and colleagues in the 1960s [5], where they observed that a subtype of cells isolated from the iliac crest's bone marrow were plastic adherent and could differentiate into chondrocytes and osteoblasts. Nowadays, MSCs are defined according to three main characteristics: 1) their ability to adhere to plastic surfaces; 2) the expression of markers like CD105, CD73 and CD90 and the lack of expression of 
CD45, CD34, CD14, CD11b, CD79 $\alpha$ or CD19 and HLA class II; and 3) their differentiation potential into osteoblasts, chondrocytes and adipocytes (multipotency potential) [6]. In addition, MSCs are also characterized by their proliferative capacity and self-renewal ability. Moreover, MSCs and their secretome exhibit trophic properties that promote regeneration in lesioned tissues [7]. This could be due to a modulation of the inflammatory responses [8] and induction of angiogenesis [9], enhancing the endogenous regeneration process. These properties associated with their differentiation potential led to the application of MSCs as a component of engineered tissue constructs, replacing diseased or damaged tissues [10].

MSCs have been mainly obtained from the bone marrow (BMMSCs) [11]. However, they can also be isolated from the adipose tissue (ASCs) [12,13], the umbilical cord [either umbilical cord itself (UC-MSCs) or its blood (UCB-MSCs)] [14] and more recently from skeletal muscles [15], dental tissues [16], synovial fluid [17], palatine tonsil [18], parathyroid gland [19], fallopian tube [20], brain [21], placental [22,23], endometrial tissues [24] and uterine cervix [25,26].

Despite the wide range of therapeutic uses for MSCs, their application to cancer treatment remains controversial. Some studies show that factors produced by MSCs can inhibit tumor growth in different animal models (including gliomas) [27,28], while others state that their use should be further investigated as they could instead promote characteristics of tumor malignancy [29]. Regarding recently-found sources of MSCs, conditioned media (CM) obtained from heat-treated amniotic fluid-derived MSCs showed a suppressive effect on ovarian tumor progression and malignancy [30]. Bu and colleagues demonstrated that human endometrial MSCs derived from menstrual blood present antitumor properties against epithelial ovarian cancer in vitro and in vivo [31]. In a very interesting work, Eiró et al. applied $\mathrm{CM}$ of MSCs from human uterine cervix (hUCESC) to breast cancer cell lines and primary tumors, inducing a reduction in cell proliferation and invasion, alterations in cell cycle and increased apoptosis [25]. The collected $\mathrm{CM}$ also affected cancer-associated fibroblasts, reverted macrophage differentiation and when applied into a xenograft mouse tumor model, reduced tumor growth and increased overall survival [25]. Nevertheless, a relevant finding about MSCs is their homing ability to tumors. This has been demonstrated in several studies and opens a door for targeted therapies.

\section{MSCs tropism towards tumors}

The tropism of MSCs towards established gliomas has already been shown by Nakamizo and colleagues in 2005 [32]. Human BMMSCs were injected into the carotid arteries of mice and were found exclusively within brain tumors, which did not happen with injected fibroblasts or U87 glioma cells that presented a more widespread distribution. In addition, BM-MSCs intracranially injected into the opposite side of the tumor were capable of migrating into the xenograft. The authors also demonstrated that CM from gliomas specifically supported BM-MSCs' invasion in an in vitro Matrigel assay, and that platelet-derived growth factor (PDGF), epidermal growth factor (EGF) and stromal cell-derived factor- $1 \alpha$ (SDF-1 $\alpha)$ were partially responsible for the enhanced MSCs invasion.

In the same direction, glioma cells engineered to secrete high levels of PDGF-BB attracted more human MSCs than low secreting gliomas [33]. Moreover, neutralizing this protein or its receptor (PDGFR-B) reduced the migratory capacity of MSCs. Similar findings were obtained in vivo, highlighting a relevant role of PDGF signaling in this innate tropism of MSCs to glioma cells. After exploring more this mechanism, Hu et al. (2013) proposed a role for vascular cell adhesion protein 1 (VCAM-1) in PDGF-BB mediated effects, since VCAM-1 neutralization inhibited BM-MSCs migration [34]. More recently, it was shown that stimulation with PDGF-BB led to an increased VCAM-1 expression by MSCs. CD44 expression was also augmented in BM-MSCs following PDGF-BB $12 \mathrm{~h}$ treatment [35]. Moreover, antibody neutralizing experiments resulted in inhibited migratory ability, as well.

Interleukin 8 (IL8) produced by glioma cell lines was demonstrated to be a chemoattractant to human UCB-MSCs, which had higher expression of IL8 receptors CXC chemokine receptors 1 and 2 (CXCR1 and CXCR2), in comparison to BM-MSCs that presented less migratory ability towards glioma [36]. Overexpression of CXCR1 further augmented the migration capacity of UCB-MSCs [37]. In a different study, rat BM-MSCs were shown to express CXCR4, besides CCR2, the respective receptors for MCP-1 and the abovementioned SDF-1 $\alpha$ [38]. These two factors were shown to be important for MSCs migration in this and other studies [39]. Overexpression of CXCR4 in human UCB-MSCs also resulted in enhanced MSCs migratory capacity [40].

Other factors that might be responsible for the attraction of MSCs to gliomas include vascular endothelial growth factor (VEGF) [41], hepatocyte growth factor (HGF) [42], transforming growth factor $\beta 1$ (TGF- $\beta 1$ ) [43] and neurotrophin 3 (NT-3) [44].

In what regards MSCs, those that express matrix metalloproteinase 1 (MMP1) at higher levels presented an increased migratory capacity [45]. Blocking the interaction between MMP1 and the protease-activated receptor 1 (PAR1) diminished MSCs migration properties. Upregulation of lymphocyte adhesion molecule (ALCAM) and N-cadherin also seems to be relevant for MSCs homing ability [46]. Additionally, the suppressor gene p27 was associated to MSCs migration towards tumors [47]. MSCs from p27null mice had decreased motility in vitro and in vivo in a C6 glioma model.

Interestingly, the tropism of MSCs towards gliomas seems to be augmented by ionizing radiation (IR) [48], with the C-C motif chemokine 2 (CCL2), present in glioma cells' secretome, playing an important role in this mechanism. This is another important aspect to consider when transplanting MSCs, which is conjugating cellbased therapy with other more conventional cancer therapies. A schematic representation of the different mechanisms proposed for MSCs tropism towards gliomas can be found in Fig. 1.

Taking into account this capacity of MSCs to migrate towards tumors, these cells have been extensively used to deliver drugs or gene therapy to gliomas. Some of these have shown important benefits in animal models of glioma and are reviewed by Stuckey and Shah (2014) [49].

MSCs engineered to express and secrete cytokines such as IL2 [28], IL12 [50], IL18 [51] or interferon $\beta$ (IFN- $\beta$ ) [52], suicide gene therapy based on cytosine deaminase (CD) [53] or herpes simplex virus thymidine kinase (HSV-tk) [54], nanoparticles [55], or tumor necrosis factor-related apoptosis inducing ligand (TRAIL) [56], are just some examples, with interesting results regarding tumor treatment, mainly in in vivo pre-clinical models.

Despite this promising data, the interaction between the "naïve" MSCs and tumor cells remains elusive.

\section{MSCs interaction with tumors}

One of the first works studying the interaction between MSCs and tumors was performed by Djouad et al., in 2003 [57]. The injection of B16 melanoma cells in mice led to tumor formation only when MSCs (from murine origin) were co-injected. At the time, it was proposed that the immunosuppressive properties of MSCs were responsible for this effect, resulting in a more protumorigenic environment [57]. 
MESENCHYMAL STEM CELLS

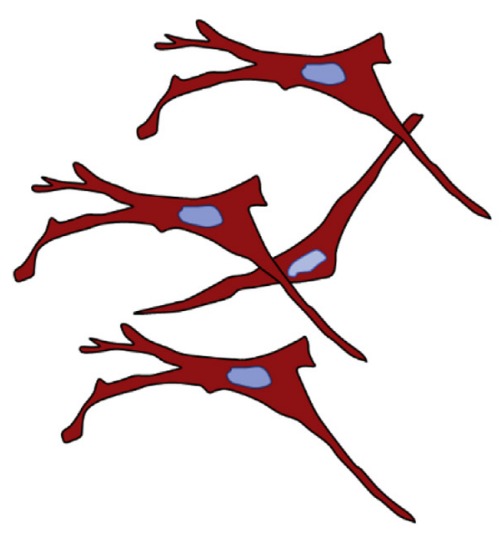

ALCAM; N-cadherin; $p 27$

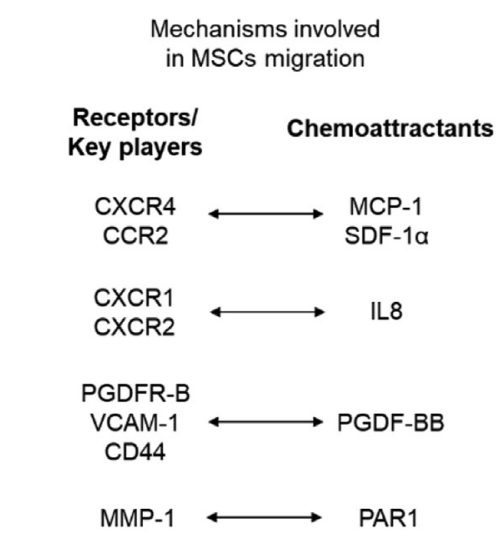

Mechanisms involved in MSCs migration

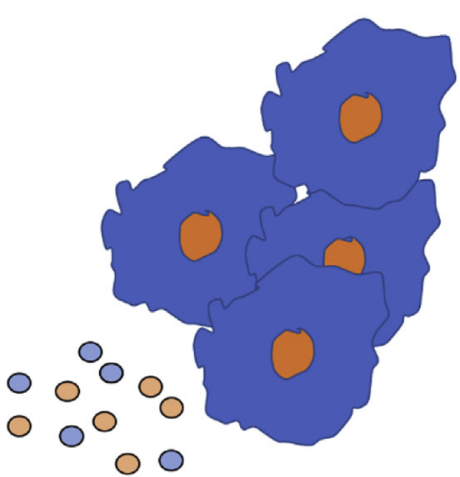

GLIOMACELLS

Other factors

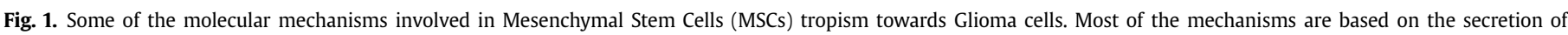
chemoattractant molecules by glioma cells and their interactions with different receptors/membrane proteins present in MSCs.

The first report that described the use of MSCs in the treatment of glioma was performed by Nakamura and colleagues [28]. Here, the authors demonstrated that MSCs derived from the bone marrow of rats could migrate toward syngeneic rat brain tumors (9 L glioma cell line) via the corpus callosum, after intracranial injection of the MSCs into the contralateral hemisphere. Interestingly, they observed that intratumoral injection of MSCs was able to decrease tumor burden and improved the overall survival (OS) of the animals, either when co-injected with tumor cells or when transplanted three days post tumor injection [28].

In 2005, Kang et al. performed one of the first studies involving the interaction between MSCs and glioma cells in vitro [58]. Using rat BM-MSCs, the authors activated these cells with several factors such as IL2, IL15 and granulocyte macrophage colony stimulating factor (GM-CSF), or different combinations of the three. Then, the co-culture of MSCs and $9 \mathrm{~L}$ rat glioma cells resulted in increased cytotoxicity to tumor cells, by a possible increase in apoptosis mechanisms [58]. Later, the same authors performed similar experiments to test human UCB-MSCs and their effect on glioma U87 cells [59]. UCB-MSCs were cytotoxic to U87 cells and this effect was more pronounced when MSCs were activated with IL2, IL15 or GM-CSF.

In another study, Dasari et al. (2010) showed that human UCBMSCs, transplanted one week after tumor inoculation, inhibited intracranial tumor growth in nude mice (injected with U251 or 5310 glioma cells), upregulating phosphatase and tensin homolog (PTEN), a tumor suppressor that negatively regulates the PI3K/Akt signaling pathway, and decreasing the levels of X-linked inhibitor of apoptosis protein (XIAP) and protein kinase B (Akt) [60]. Similar findings were obtained in vitro, with UCB-MSCs inhibiting different glioma cell lines migration and wound healing capacity [60].

An interesting work from Chien et al. (2011) labeled human BMMSCs for in vivo magnetic resonance imaging (MRI) [39]. MSCs injected two weeks after tumor inoculation (with U87 cells), at the contralateral brain hemisphere, led to glioma shrinkage as measured by signal intensity. This was later confirmed by histological analysis of the tumor tissues [39].

Still in what regards anti-tumoral effects, Ho et al. (2013) saw that human BM-MSCs inhibited the growth of the glioma cell line $\Delta$ Gli36 and patient-derived gliomas, after direct in vitro co-culture experiments [61]. This was seen by a reduction of the number of cells after flow cytometry analysis, in comparison to glioma monocultures or co-cultures with normal-human astrocytes. The in vivo subcutaneous co-administration of MSCs and $\Delta$ Gli36 cells into nude mice resulted in significant reduction of tumor volume and vascular density. After in vitro cultures using endothelial progenitor cells (EPCs) or human umbilical vein endothelial cells (HUVEC) and the MSCs' secretome, it was observed an impaired capacity of EPCs to form endothelial tubes. Therefore, the authors hypothesized that BM-MSCs suppress tumor angiogenesis through the release of anti-angiogenic factors. Moreover, after gene and protein expression analysis, alterations in PDGF/PDGFR axis seems to be relevant for the antiangiogenic effects observed [61].

More recently, Pacioni et al. (2017) transplanted human ASCs and BM-MSCs, either by direct inoculation onto the tumor and adjacent tissues [orthotopic GBM or Glioma Stem Cells (GSCs) xenografts] or by systemic injections [62]. Intracerebral injection of ASCs resulted in improved survival of rats with U87 xenografts, with reduced tumor growth, proliferation and microvascular density. ASCs and BM-MSCs injected systemically were additionally capable of homing towards tumor xenografts [62].

However, there are also studies in which MSCs apparently do not show any effect over gliomas or can even promote tumorigenic features. Bexell et al. (2009) evaluated the role of transplanted rat BM-MSCs in experimental gliomas (N29 and N32 glioma cell lines) [63]. Besides confirming the specific migration of MSCs to tumors, the authors showed that intratumoral cellular transplantation one day after tumor inoculation, did not influence the survival of tumor-bearing rats. Interestingly, they suggest that MSCs resembled pericyte-like cells, since they integrated into tumor vessel walls and expressed pericyte markers such as $\alpha$-smooth muscle actin, neuron-glia 2 and PDGF receptor- $\beta$ [63].

Roger et al. (2012) used a cell line of MSCs, the human marrow isolated adult multilineage inducible (MIAMI) cells, to target a U87 orthotopic glioma model and no influence was seen in tumor growth rate [64]. However, the authors hypothesized that the results may be glioma-dependent, because MIAMI cells could in fact induce proliferation of other glioma cell lines in vitro. Moreover, a subset of MIAMI cells was found to be in a proliferative state in the U87 tumor microenvironment [64]. A similar effect was seen by Breznik et al. (2017) [65]. Human BM-MSCs inhibited U87 cells invasion in vitro and in vivo, while on the other hand stimulated U373 invasion capacity. Inherent differences in gene expression, namely in genes responsible for the response to growth factors 
(overexpressed in U373 cells) might explain the differences observed between the two GBM cell lines [65].

The pro- or anti-tumoral effect of MSCs on gliomas might depend as well on the source of MSCs. Indeed, it is known that the secretome of MSCs isolated from different sources varies significantly [66]. As demonstrated by Akimoto et al. (2013), human UCBMSCs and ASCs interactions with gliomas were different [67]. In in vitro co-cultures, UCB-MSCs inhibited primary GBM tumor cells growth and caused apoptosis, while ASCs promoted cellular growth. TRAIL was more expressed in UCB-MSCs, and it is known its involvement in apoptosis mechanisms [68,69]. In contrast, more vascularized tumors were formed when ASCs were subcutaneously co-transplanted with primary glioma cells into mice [67].

Some works also suggest that MSCs co-cultured with tumor cells might undergo malignant transformation. Liu et al. (2012) have seen that rat BM-MSCs in indirect cultures with $\mathrm{C} 6$ glioma cells presented decreased wild type p53 and increased expression of mutant p53 and mdm2 [70]. When transplanted subcutaneously into immunodeficient-mice, MSCs developed a large tumor at the injection site within eight weeks. The authors showed that IL6 and STAT3 signaling were important factors in this mechanism [71].

The in vivo interactions between different glioma cells and MSCs from various sources are summarized in Table 1. In general, a substantial part of the studies shows a beneficial effect of MSCs over glioma-derived tumors. However, some important aspects are worth to highlight. For instance, similar U87 xenograft mice models treated with human MSCs presented different outcomes most likely due to the different source of the MSCs used (BM-MSCs vs MIAMI cells) [39,64]. Furthermore, in another study [65], different xenografts (U87 vs U373) in zebrafish embryos had different results after implantation of the same human BM-MSCs. Finally, in rat syngeneic glioma models, different rat glioma cell lines (9 L vs N29/ N32) can also impact on the outcome following rat BM-MSCs application $[28,63]$. All of these show the importance of the glioma cell line used, its origin (human or rat) and also the source of MSCs under study.

\section{MSCs secretome and gliomas}

A relatively small number of studies have focused on the factors secreted by MSCs and their effects on glioma development and growth. Being MSCs a population of cells with a high paracrine signaling component, it is crucial to better understand how this mechanism could affect gliomas.

In 2014, Ma et al. explored in vitro the molecular mechanisms by which human UC-MSCs inhibit the growth of C6 glioma cells [72]. Increased levels of dickkopf-1 (DKK1) were observed in UC-MSCs $\mathrm{CM}$, and the cell inhibitory effect was DKK1-concentrationdependent. Moreover, the use of anti-DKK1 antibodies attenuated the inhibition on C6 cells growth. By downregulating or upregulating DKK1, the CM from UC-MSCs lost or gained respectively abilities to regulate Wnt signaling in C6 cells. In turn, tumor cells exposed to UC-MSCs CM presented a downregulation in $\beta$-catenin and c-Myc expression [72].

Using the glioma cell line U251 as a model, Yang et al. (2014) showed that both human ASCs and UC-MSCs CM significantly affected tumor cells' proliferation, migration and invasion [73]. This was accompanied by an increase in apoptosis (more significant with UC-MSCs CM), together with upregulation of caspase- 3 and caspase-9 and downregulation of antiapoptotic genes, such as survivin and XIAP. Curiously, a partial differentiation of U251 cells into astrocyte-like cells (increased GFAP expression) was also observed after treatment with both MSCs' CM [73].

Relevant work from Kološa et al. (2015) assessed the effects of MSCs CM on a particular subset of tumor cells, with stem-like properties (GBM stem-like cells - GSLCs) [74]. CM from human BM-MSCs and UC-MSCs were tested on four different GSLC lines. Cell cycle arrest was induced, with decreased expression of cyclin D1. The factors secreted by MSCs also induced senescence, deregulating a total of 13 genes associated with this process, in one of the GSLC lines. Cell cycle inhibitors p21 and p16, which can trigger senescence, were upregulated in all four lines after CM exposure. Increased sensitivity against the chemotherapeutic drug temozolomide was observed, possibly by inducing differentiation of GSLCs (upregulation of vimentin and GFAP, with concomitant downregulation of Sox-2 and Notch-1) [74]. These results could be of the utmost importance, since these cells are highly resistant to conventional chemo- and radiotherapy regimens, being often responsible for recurrence in gliomas.

In an interesting work, Del Fattore et al. (2015) analyzed the effect of extracellular vesicles (EVs) secreted by human MSCs derived from various tissue-sources, on U87 cells [75]. Using $48 \mathrm{~h}$ CM from ASCs, BM-MSCs and UC-MSCs, EVs were isolated and applied to U87 cultures. It was demonstrated that EVs were internalized by glioma cells and their effects were different according to their source. EVs from BM- and UC-MSCs decreased cell proliferation and induced apoptosis. On the contrary, ASCs-secreted EVs increased U87 cell proliferation and had no effect on apoptosis. Curiously, the supernatant of all MSCs CM (containing the secreted non-vesicular fraction) did not induce any change on glioma cells, suggesting that the effects observed were specific to the secreted EVs, when isolated from the secretome as a whole [75]. EVs are produced and released by the outer membrane of several types of cells, including MSCs (MSCs-EVs). These MSCs-EVs are a heterogeneous population of vesicles with a diameter of $40-1000 \mathrm{~nm}$ that mainly include exosomes, apoptotic bodies, and microvesicle particles. Several studies have been describing that MSCs-EVs may have different effects on tumor growth, metastasis, and drug response. In fact, some studies demonstrated that these MSCs-EVs have a tumor-supportive effect, while others showed the opposite [recently reviewed in Lee et al. (2017) [76] and Zhang et al., 2017 [77]].

Chistyakova and Poljanskaya (2015) tested MSCs from human fetal tissues, either from fetal bone marrow or fetal muscle [78]. CM from both fetal MSCs per se did not influence the proliferation of U251 and A172 glioma cells. However, CM collected from cocultures of fetal MSCs with U251 cells, led to interesting results: CM from early time-points of co-culture (3-9 days) stimulated glioma cells proliferation, while $\mathrm{CM}$ obtained from later timepoints (15-21 days) resulted in inhibited cellular proliferation [78]. This study raised two relevant questions: first, it is important to explore changes in secretome when MSCs are co-cultured with tumor cells; second, the prolonged co-culture of MSCs with glioma cells led to a shift from a pro-tumorigenic to a non-tumorigenic CM, which highlights the importance of further testing the timing of $\mathrm{CM}$ collection.

Another interesting finding was the contrasting effect observed by Bajetto et al. (2017) regarding direct cell-cell contact and secretome-mediated effects [79]. Human UC-MSCs cultured in direct contact with GBM CSCs inhibited their proliferation, while the factors secreted by UC-MSCs increased CSCs proliferation rate through transient ERK1/2 and Akt phosphorylation/activation. Among those factors IL8, CXCL1, CXCL5 and IL6 were present. In addition, activation of the CXC chemokine receptor 2 (expressed in GBM cells) seems to be crucial for the effects observed [79].

A possible explanation could reside on the fact that MSCs growing in monocultures might secrete a different set of factors and cytokines, which most likely would be different if they were pre-conditioned by the presence of tumor cells, either their factors or the cells themselves. Nevertheless, this study focusses on in vitro 
Table 1

Different in vivo models of glioma and treatments with MSCs from various sources.

\begin{tabular}{|c|c|c|c|c|c|c|c|}
\hline Tumor cell line(s) & $\begin{array}{l}\text { Tissue } \\
\text { source }\end{array}$ & $\begin{array}{c}\text { Animal } \\
\text { model }\end{array}$ & $\begin{array}{c}\text { Tumor } \\
\text { inoculation }\end{array}$ & MSCs & $\begin{array}{c}\text { Mode of } \\
\text { administration }\end{array}$ & Main Outcomes & Ref \\
\hline \multirow{5}{*}{ U87 } & $\begin{array}{l}\text { Human } \\
\text { glioma }\end{array}$ & Mice & IC injection & $\begin{array}{l}\text { Human BM- } \\
\text { MSCs }\end{array}$ & $\begin{array}{l}\text { IC injection } \\
\text { (at contralateral } \\
\text { hemisphere), } 2 \mathrm{w} \text { post- } \\
\text { tumor inoculation }\end{array}$ & $\begin{array}{l}\text { Tumor shrinkage detected by MRI and } \\
\text { H\&E histology }\end{array}$ & (39) \\
\hline & $/ /$ & // & /l & $\begin{array}{l}\text { Human } \\
\text { MIAMI } \\
\text { cells }\end{array}$ & $\begin{array}{l}\text { Intratumoral injection, } \\
6 \mathrm{~d} \text { post-tumor } \\
\text { inoculation }\end{array}$ & No differences in OS & (64) \\
\hline & $/ /$ & Rat & $/ 1$ & $\begin{array}{c}\text { Human } \\
\text { ASCs }\end{array}$ & $\begin{array}{l}\text { Injection into adjacent } \\
\text { tumor tissues, right after } \\
\text { tumor inoculation }\end{array}$ & $\begin{array}{c}\text { Improved OS; Reduced tumor growth, } \\
\text { decreased cell proliferation and } \\
\text { angiogenesis }\end{array}$ & \multirow{2}{*}{$(62)$} \\
\hline & $/ /$ & $/ /$ & // & $\begin{array}{l}\text { Human } \\
\text { ASCs and } \\
\text { BM-MSCs }\end{array}$ & $\begin{array}{l}\text { Systemic injections, } 2 \mathrm{w} \\
\text { post-tumor inoculation }\end{array}$ & $\begin{array}{l}\text { Homing capacity of MSCs into tumors } \\
\text { with no other outcomes reported }\end{array}$ & \\
\hline & // & $\begin{array}{c}\text { Zebrafish } \\
\text { embryo }\end{array}$ & // & $\begin{array}{l}\text { Human BM- } \\
\text { MSCs }\end{array}$ & $\begin{array}{l}\text { Co-injections of MSCs } \\
\text { with tumor cells }\end{array}$ & $\begin{array}{l}\text { Reduced tumor cell proliferation and } \\
\text { invasion }\end{array}$ & (65) \\
\hline GSCs & $/ /$ & Rat & // & $\begin{array}{c}\text { Human } \\
\text { ASCs }\end{array}$ & $\begin{array}{l}\text { Injection into the tumor, } \\
16 \mathrm{w} \text { post-tumor } \\
\text { inoculation }\end{array}$ & $\begin{array}{l}\text { Reduced tumor cell densities; decreased } \\
\text { cell proliferation }\end{array}$ & $(62)$ \\
\hline $\begin{array}{l}\text { U251 and } 5310 \\
\text { (patient derived } \\
\text { glioma) }\end{array}$ & $\begin{array}{l}\text { Human } \\
\text { glioma }\end{array}$ & Mice & IC injection & $\begin{array}{l}\text { Human } \\
\text { UCB-MSCs }\end{array}$ & $\begin{array}{l}\text { IC injection } \\
\text { (contralateral } \\
\text { hemisphere), 1w post- } \\
\text { tumor inoculation }\end{array}$ & $\begin{array}{c}\text { Tumor growth inhibition; PTEN } \\
\text { upregulation; XIAP and Akt } \\
\text { downregulation }\end{array}$ & $(60)$ \\
\hline U373 & $/ /$ & $\begin{array}{l}\text { Zebrafish } \\
\text { embryo }\end{array}$ & $/ /$ & $\begin{array}{l}\text { Human BM- } \\
\text { MSCs }\end{array}$ & $\begin{array}{l}\text { Co-injections of MSCs } \\
\text { with tumor cells }\end{array}$ & $\begin{array}{l}\text { Reduced cell tumor proliferation but } \\
\text { enhanced cell invasion }\end{array}$ & $(65)$ \\
\hline$\Delta$ Gli36 & /l & Mice & $\begin{array}{l}\text { Subcutaneous } \\
\text { inoculation }\end{array}$ & 1000 & ง & $\begin{array}{c}\text { Reduced tumor volume and vascular } \\
\text { density; Reduced expression of IL- } 1 \beta \text {, } \\
\text { cathepsin B, PDGF-BB, PDGFR- } \beta \text { and } \\
\text { pAkt }\end{array}$ & (61) \\
\hline $\begin{array}{l}\text { GBM\#1 and } \\
\text { GBM\#12 (patient } \\
\text { derived gliomas) }\end{array}$ & $/ /$ & // & /I & $\begin{array}{l}\text { Human } \\
\text { UCB-MSCs } \\
\text { and ASCs }\end{array}$ & // & $\begin{array}{l}\text { Reduced tumor weights and increased } \\
\text { apoptosis for hUCB-MSCs; } \\
\text { Increased tumor weights, higher } \\
\text { vascularization for hASCs }\end{array}$ & (67) \\
\hline 9L & $\begin{array}{l}\text { Rat } \\
\text { glioma }\end{array}$ & Rat & IC injection & $\begin{array}{l}\text { Rat BM- } \\
\text { MSCs }\end{array}$ & $\begin{array}{l}\text { Co-injection of MSCs } \\
\text { with tumor cells or } \\
\text { intratumoral injection }\end{array}$ & Improved OS; Reduced tumor volumes & (28) \\
\hline $\mathrm{N} 29$ and N32 & // & $/ /$ & /I & // & $\begin{array}{l}\text { Intratumoral injections, } \\
1 \mathrm{~d} \text { after tumor } \\
\text { inoculation; or Systemic } \\
\text { injections } 14 \mathrm{~d} \text { post } \\
\text { tumor inoculaton }\end{array}$ & $\begin{array}{c}\text { No alterations in OS and tumor } \\
\text { microvasculature; } \\
\text { No MSCs were found in tumors after } \\
\text { systemic injections }\end{array}$ & (63) \\
\hline
\end{tabular}

Green: overall antitumor effects; Yellow: no effect; Red: contrasting or pro-tumoral effects.

IC - Intracerebral; MIAMI - marrow-isolated adult multilineage inducible; OS - overall survival; GSCs - glioma stem cells.

findings, which does not reflect the tumor microenvironment effect.

Iser et al. (2016) evaluated whether CM from ASCs (rat origin) could have an effect on rat C6 glioma cells [80]. Even though cell viability, cell cycle and growth rate were not altered, there was an increase in the migratory capacity of C6 cells. Furthermore, CM treatment led to reduced cell adhesion and changes in their morphology. At the molecular level, an upregulation of epithelialto-mesenchymal transition (EMT)-markers was observed, namely vimentin, MMP2 and NRAS. EMT is a process often associated to cancer development and progression. One of the most important differences of this study is the origin of ASCs, which are from rat origin. Nevertheless, ASCs CM presented a more pro-tumorigenic effect, which goes along with Del Fattore and colleagues [75] data for human ASCs CM.

Also using the secretome of human ASCs, Onzi et al. (2016) [81] demonstrated that U87 GBM cells presented increased migration, even though there were no alterations in cell proliferation, response to temozolomide and sphere-forming capacity.

Our group has also explored the impact of human umbilical cord perivascular cells (HUCPVCs) secretome on two GBM cell lines, U251 and SNB-19 [82]. Tumor cells exposed to this secretome presented significantly higher cellular viability, proliferation and migration in vitro, in comparison to cells exposed to control media. Moreover, using the in vivo chicken chorioallantoic membrane (CAM) assay, HUCPVCs CM induced tumor growth and increased the number of vessels. Molecules such as CCL2, periostin (POSTN) or IL6 were among the factors secreted by HUCPVCs, which have been described to modulate glioma cells migration and adhesion, invasion, or even angiogenesis, important hallmarks of glioma development. However, it is important to notice that, as addressed before, the secretome of MSCs changes when these cells interact directly with tumor cell lines. Thus, it would be important in future studies to further understand how this particular stem cells population interacts with GBM cell lines and their microenvironment.

Overall, the results obtained using MSCs CM in glioma cells are distinct and variations in protocols might explain these differences. One important aspect to consider is the use of serum in the media for $\mathrm{CM}$ collection, which seems to be associated with a more protumorigenic effect, even though there are some exceptions (Table 2). Nevertheless, the proteins and factors present in each secretome should be identified and quantified in order to better dissect each secretome effect.

The secretome of MSCs has been characterized by our group and others $[13,83,84]$, and numerous proteins and small vesicles can be found. Many of them with potential pro- or anti-tumorigenic capacity. So far, several signaling molecules secreted by MSCs have been described to be involved in different biological processes, such as ECM remodeling, angiogenesis, mitogenic remodeling, apoptosis inhibition, and tumor aggressiveness, among others [85-87]. MSCsecreted proteins are capable of coordinating survival, migration, proliferation, and differentiation responses on healthy tissues and cancer cells through the activation of many signaling cascades. Additionally, the secretome of MSCs also presents proangiogenic 
Table 2

In vitro studies using the secretome of MSCs in different glioma cell lines.

\begin{tabular}{|c|c|c|c|c|c|c|}
\hline MSCs & Passage & Serum conditions & $\begin{array}{c}\text { Time of } \mathrm{CM} \\
\text { collection }\end{array}$ & $\begin{array}{l}\text { Glioma } \\
\text { cell lines }\end{array}$ & Main Outcomes & Ref \\
\hline Human ASCs & N/A & $5 \%$ FBS & $48 \mathrm{~h}$ & U87 & $\begin{array}{l}\text { Increased migration; } \\
\text { No alterations: sphere-forming capacity, proliferation, response to } \\
\text { TMZ }\end{array}$ & $(81)$ \\
\hline Rat ASCs & P4-P10 & $10 \%$ FBS & $24 \mathrm{~h}, 48 \mathrm{~h}$ & $\mathrm{C} 6$ & $\begin{array}{l}\text { Increased migration, changes in cell morphology, reduced } \\
\text { adhesion, upregulation of EMT markers; } \\
\text { No alterations: cell viability, cell cycle, growth rate }\end{array}$ & $(80)$ \\
\hline $\begin{array}{l}\text { Human ASCs, } \\
\text { UC-MSCs }\end{array}$ & P3 & $0 \%$ FBS & $48 \mathrm{~h}$ & $\mathrm{U} 251$ & $\begin{array}{c}\text { Cell growth inhibition, induced apoptosis, upregulation of } \\
\text { apoptotic genes/downregulation of antiapoptotic genes, induced } \\
\text { differentiation of cells }\end{array}$ & (73) \\
\hline $\begin{array}{l}\text { Human ASCs, } \\
\text { BM-MSCs, } \\
\text { UC-MSCs }\end{array}$ & P3 & $\begin{array}{l}10 \% \text { FBS } \\
\text { (ultracentrifuged) }\end{array}$ & $48 \mathrm{~h}$ & U87 & $\begin{array}{l}\text { ASCs-derived EVs promoted cell proliferation; } \\
\text { BM-MSCs and UC-MSCs-derived EVs decreased cell } \\
\text { proliferation, induced apoptosis }\end{array}$ & $(75)$ \\
\hline $\begin{array}{l}\text { Human BM- } \\
\text { MSCs, UC- } \\
\text { MSCs }\end{array}$ & N/A & $0 \%$ FBS & $24 \mathrm{~h}$ & GSLCs & $\begin{array}{l}\text { Cell cycle arrest, increased senescence and sensitivity against } \\
\qquad \mathrm{TMZ}\end{array}$ & (74) \\
\hline $\begin{array}{l}\text { Human UC- } \\
\text { MSCs }\end{array}$ & P3-P5 & $10 \%$ FBS & $\begin{array}{l}\text { Upon } 80-90 \% \\
\text { confluence }\end{array}$ & $\mathrm{C} 6$ & Inhibited cell growth, cell cycle arrest & $(72)$ \\
\hline $\begin{array}{l}\text { Human UC- } \\
\text { MSCs }\end{array}$ & P2-P6 & $0 \%$ FBS & $48 \mathrm{~h}$ & $\mathrm{CSCs}$ & $\begin{array}{l}\text { Increased proliferation rate, transient ERK1/2 and Akt } \\
\text { activation/phosphorylation }\end{array}$ & (79) \\
\hline $\begin{array}{l}\text { Human } \\
\text { HUCPVCs }\end{array}$ & P6 & $\begin{array}{l}0 \% \text { FBS at } \\
\text { collection, } 1 \% \text { FBS } \\
\text { added posteriorly }\end{array}$ & $48 \mathrm{~h}$ & $\begin{array}{l}\mathrm{U} 251, \\
\text { SNB-19 }\end{array}$ & $\begin{array}{l}\text { Higher cell viability, proliferation and migration, } \\
\text { Increased tumors in CAM assay and more blood vessels }\end{array}$ & $(82)$ \\
\hline $\begin{array}{l}\text { Human fetal } \\
\text { BM-MSCs, } \\
\text { muscle MSCs }\end{array}$ & P6-P8 & $10 \%$ FCS & $48 \mathrm{~h}, 72 \mathrm{~h}$ & $\begin{array}{l}\mathrm{U} 251, \\
\mathrm{~A} 172\end{array}$ & $\begin{array}{c}\text { MSCs CM had no effect on glioma cells } \\
\text { CM from MSCs co-cultures with U251 cells (3-9 days) led to } \\
\text { increased proliferation; CM from co-cultures (15-21 days) had } \\
\text { inhibitory effects }\end{array}$ & $(78)$ \\
\hline
\end{tabular}

Green: overall antitumor effects; Red: contrasting or pro-tumoral effects.

$\mathrm{CM}$ - conditioned medium; CSCs - cancer stem cells; EMT - epithelial to mesenchymal transition; EVs - extracellular vesicles; FBS - fetal bovine serum; FCS - fetal calf serum; GSLCs - glioblastoma stem-like cells (NCH421k, NCH644, NIB26, NIB50); TMZ - temozolomide.

effects, ECM components and proteins that regulate its composition. Finally, MSCs secrete a variety of chemoattractant molecules capable of recruiting diverse cell types, such as immune and progenitor cells (including MSCs themselves).

Besides the ones already mentioned, others such as periostin [88], semaphorin-7A [89], testican-1 [90] or neuropilin-2 [91] are also present in MSCs secretome and known to influence tumor behavior. A summary of the most common factors present in MSCs secretome that can influence glioma cells is described in Fig. 2. Further characterization of secretome composition is essential in order to fully understand the potential benefits or drawbacks of MSCs application to treat human gliomas.

\section{Conclusions}

Several factors might explain the differences observed in the

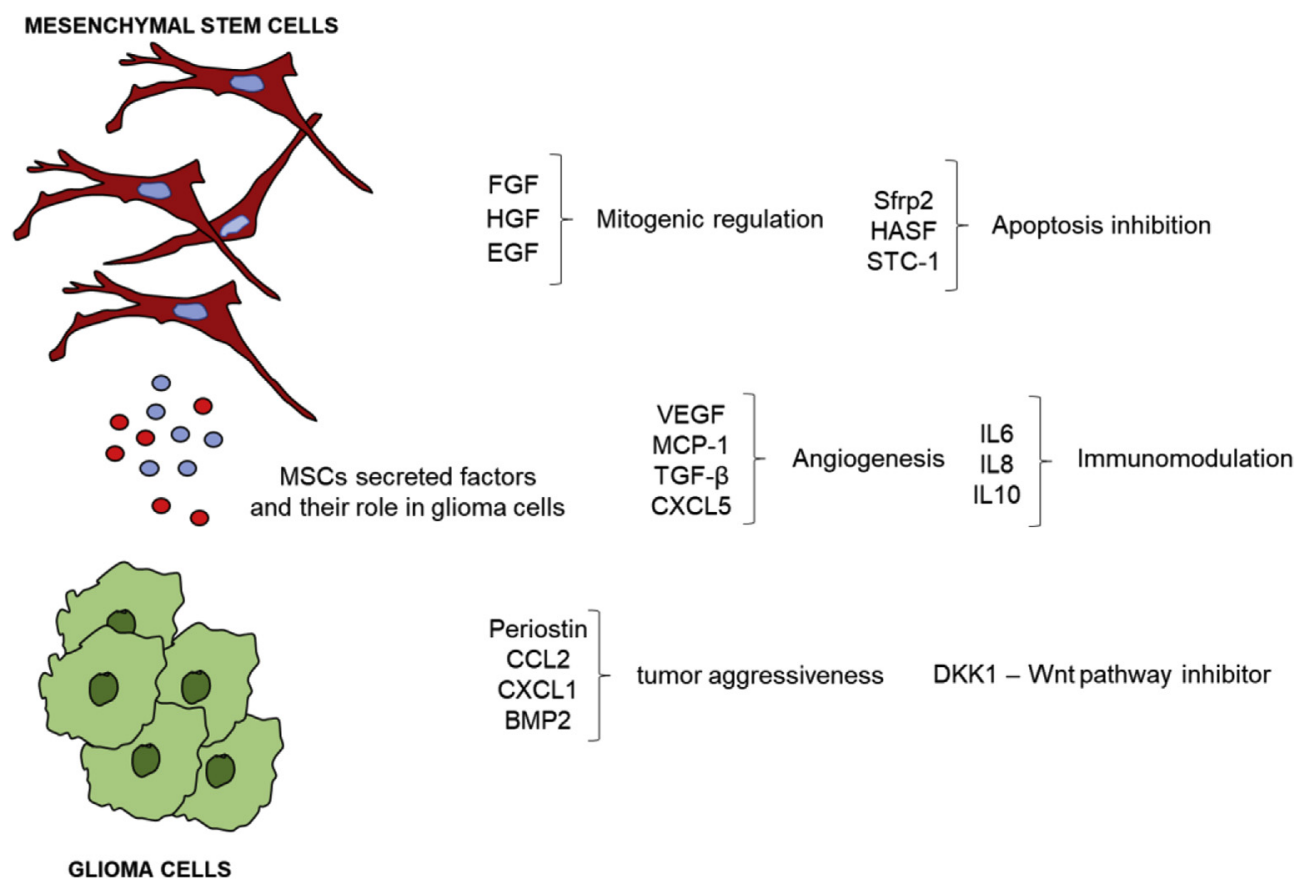

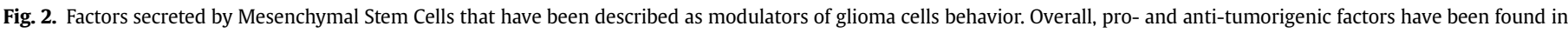
MSCs secretome. 
results described so far, in the interaction between MSCs and tumor cells. Variations in protocols are one of the main reasons. Indeed, different periods of CM collection or different media compositions (particularly serum percentage) are just some examples. Adding to this, MSCs' source, and even differences among donors or batches of isolated cells can influence the outcome of each experiment. In what concerns in vivo models, there is a big variation. Excluding the differences introduced by cell culturing and animal models, the mode how the glioma is induced or the timing for MSCs transplantation often differs from study to study. There is also a lack of in vivo proof-of-concept assays, testing MSCs' secretome effects on gliomas. Due to its potential of reducing the number of cells needed to possibly obtain similar findings, while avoiding problems with immune rejection, it is necessary to further test this approach. Overall, MSCs could present an interesting potential as a cancer treatment strategy, particularly when engineered to deliver antitumor cargos. Their homing ability towards tumor tissues is a critical feature for their application, however MSCs interactions with tumor cells still needs to be further explored. Determining the optimal source for MSCs, the best culture conditions, or even the most appropriate timing for treatment is essential. In addition, the development of improved cancer models, both in vitro and in vivo, is of the utmost importance to test this. The understanding of this complex interaction between gliomas and MSCs is a key aspect for future applications in humans and to improve the prognosis of these patients.

\section{Acknowledgments}

Portuguese Foundation for Science and Technology: predoctoral fellowship to E.D. Gomes (SFRH/BD/103075/2014) and J. Vieira de Castro (SFRH/BD/88121/2012); FCT Investigator Starting Grant to B.M. Costa (IF/00601/2012); FCT Investigator Development Grant to A.J. Salgado (IF/00111/2013). This article has been developed under the scope of the project NORTE-01-0145-FEDER000023, supported by the Northern Portugal Regional Operational Programme (NORTE 2020), under the Portugal 2020 Partnership Agreement, through the European Regional Development Fund (FEDER). This work has also been funded by FEDER funds, through the Competitiveness Factors Operational Programme (COMPETE), and by National funds, through the Foundation for Science and Technology (FCT), under the scope of the project POCI-01-0145FEDER-007038.

\section{References}

[1] M.L. Goodenberger, R.B. Jenkins, Genetics of adult glioma, Cancer genetics 205 (12) (2012) 613-621

[2] Q.T. Ostrom, H. Gittleman, L. Stetson, S.M. Virk, J.S. Barnholtz-Sloan, Epidemiology of gliomas, Canc. Treat Res. 163 (2015) 1-14.

[3] R. Stupp, et al., Radiotherapy plus concomitant and adjuvant temozolomide for glioblastoma, N. Engl. J. Med. 352 (10) (2005) 987-996.

[4] M.L. Cruceru, et al., Signal transduction molecule patterns indicating potential glioblastoma therapy approaches, OncoTargets Ther. 6 (2013) 1737-1749.

[5] A.J. Friedenstein, et al., Precursors for fibroblasts in different populations of hematopoietic cells as detected by the in vitro colony assay method, Exp. Hematol. 2 (2) (1974) 83-92.

[6] M. Dominici, et al., Minimal criteria for defining multipotent mesenchyma stromal cells. The International Society for Cellular Therapy position statement, Cytotherapy 8 (4) (2006) 315-317.

[7] A.I. Caplan, Adult mesenchymal stem cells for tissue engineering versus regenerative medicine, J. Cell. Physiol. 213 (2) (2007) 341-347.

[8] K. Le Blanc, C. Tammik, K. Rosendahl, E. Zetterberg, O. Ringden, HLA expression and immunologic properties of differentiated and undifferentiated mesenchymal stem cells, Exp. Hematol. 31 (10) (2003) 890-896.

[9] Y.L. Tang, et al., Autologous mesenchymal stem cell transplantation induce VEGF and neovascularization in ischemic myocardium, Regul. Pept. 117 (1) (2004) 3-10.

[10] A.I. Caplan, S.P. Bruder, Mesenchymal stem cells: building blocks for molec ular medicine in the 21st century, Trends Mol. Med. 7 (6) (2001) 259-264.
[11] F.P. Barry, J.M. Murphy, Mesenchymal stem cells: clinical applications and biological characterization, Int. J. Biochem. Cell Biol. 36 (4) (2004) 568-584.

[12] P.A. Zuk, et al., Human adipose tissue is a source of multipotent stem cells, Mol. Biol. Cell 13 (12) (2002) 4279-4295.

[13] A.J. Salgado, R.L. Reis, N.J. Sousa, J.M. Gimble, Adipose tissue derived stem cells secretome: soluble factors and their roles in regenerative medicine, Curr. Stem Cell Res. Ther. 5 (2) (2010) 103-110.

[14] O.K. Lee, et al., Isolation of multipotent mesenchymal stem cells from umbilical cord blood, Blood 103 (5) (2004) 1669-1675.

[15] W.M. Jackson, L.J. Nesti, R.S. Tuan, Potential therapeutic applications of muscle-derived mesenchymal stem and progenitor cells, Expet Opin. Biol. Ther. 10 (4) (2010) 505-517.

[16] G.T. Huang, S. Gronthos, S. Shi, Mesenchymal stem cells derived from dental tissues vs. those from other sources: their biology and role in regenerative medicine, J. Dent. Res. 88 (9) (2009) 792-806.

[17] E.A. Jones, et al., Enumeration and phenotypic characterization of synovial fluid multipotential mesenchymal progenitor cells in inflammatory and degenerative arthritis, Arthritis Rheum. 50 (3) (2004) 817-827.

[18] S. Janjanin, et al., Human palatine tonsil: a new potential tissue source of multipotent mesenchymal progenitor cells, Arthritis Research \& Therapy 10 (4) (2008) R83.

[19] Y.R. Shih, T.K. Kuo, A.H. Yang, O.K. Lee, C.H. Lee, Isolation and characterization of stem cells from the human parathyroid gland, Cell Proliferation 42 (4) (2009) 461-470.

[20] T. Jazedje, et al., Human fallopian tube: a new source of multipotent adult mesenchymal stem cells discarded in surgical procedures, J. Transl. Med. 7 (2009) 46.

[21] G. Paul, et al., The adult human brain harbors multipotent perivascular mesenchymal stem cells, PLoS One 7 (4) (2012) e35577.

[22] Y. Fukuchi, et al., Human placenta-derived cells have mesenchymal stem/ progenitor cell potential, Stem Cell. 22 (5) (2004) 649-658.

[23] L. Li, et al., Characteristics of human amniotic fluid mesenchymal stem cells and their tropism to human ovarian cancer, PLoS One 10 (4) (2015) e0123350.

[24] K.E. Schwab, P. Hutchinson, C.E. Gargett, Identification of surface markers for prospective isolation of human endometrial stromal colony-forming cells, Hum. Reprod. 23 (4) (2008) 934-943.

[25] N. Eiro, et al., Potential therapeutic effect of the secretome from human uterine cervical stem cells against both cancer and stromal cells compared with adipose tissue stem cells, Oncotarget 5 (21) (2014) 10692-10708.

[26] J. Schneider, N. Eiro, R. Perez-Fernandez, A. Martinez-Ordonez, F. Vizoso, Human uterine cervical stromal stem cells (hUCESCs): why and how they exert their antitumor activity, Cancer Genomics Proteomics 13 (5) (2016) $331-337$.

[27] G.J. Maestroni, E. Hertens, P. Galli, Factor(s) from nonmacrophage bone marrow stromal cells inhibit Lewis lung carcinoma and B16 melanoma growth in mice, Cell. Mol. Life Sci. : CM 55 (4) (1999) 663-667.

[28] K. Nakamura, et al., Antitumor effect of genetically engineered mesenchymal stem cells in a rat glioma model, Gene Therapy 11 (14) (2004) 1155-1164.

[29] W. Zhu, et al., Mesenchymal stem cells derived from bone marrow favor tumor cell growth in vivo, Exp. Mol. Pathol. 80 (3) (2006) 267-274.

[30] J.A. Cho, et al., Hyperthermia-treated mesenchymal stem cells exert antitumor effects on human carcinoma cell line, Cancer 115 (2) (2009) 311-323.

[31] S. Bu, et al., Human endometrial mesenchymal stem cells exhibit intrinsic anti-tumor properties on human epithelial ovarian cancer cells, Sci. Rep. 6 (2016), 37019.

[32] A. Nakamizo, et al., Human bone marrow-derived mesenchymal stem cells in the treatment of gliomas, Canc. Res. 65 (8) (2005) 3307-3318.

[33] N. Hata, et al., Platelet-derived growth factor BB mediates the tropism of human mesenchymal stem cells for malignant gliomas, Neurosurgery 66 (1) (2010) 144-156, discussion 156-147.

[34] Y. Hu, P. Cheng, J.C. Ma, Y.X. Xue, Y.H. Liu, Platelet-derived growth factor BB mediates the glioma-induced migration of bone marrow-derived mesenchymal stem cells by promoting the expression of vascular cell adhesion molecule-1 through the PI3K, P38 MAPK and NF-kappaB pathways, Oncol. Rep. 30 (6) (2013) 2755-2764.

[35] Q. Yin, et al., CD44 promotes the migration of bone marrow-derived mesenchymal stem cells toward glioma, Oncology letters 11 (4) (2016) 2353-2358.

[36] D.S. Kim, et al., Overexpression of CXC chemokine receptors is required for the superior glioma-tracking property of umbilical cord blood-derived mesenchymal stem cells, Stem Cells and Development 18 (3) (2009) 511-519.

[37] S.M. Kim, et al., CXC chemokine receptor 1 enhances the ability of human umbilical cord blood-derived mesenchymal stem cells to migrate toward gliomas, Biochem. Biophys. Res. Commun. 407 (4) (2011) 741-746.

[38] F. Xu, et al., Chemokines mediate mesenchymal stem cell migration toward gliomas in vitro, Oncol. Rep. 23 (6) (2010) 1561-1567.

[39] L.Y. Chien, et al., In vivo magnetic resonance imaging of cell tropism, trafficking mechanism, and therapeutic impact of human mesenchymal stem cells in a murine glioma model, Biomaterials 32 (12) (2011) 3275-3284.

[40] S.A. Park, et al., CXCR4-transfected human umbilical cord blood-derived mesenchymal stem cells exhibit enhanced migratory capacity toward gliomas, Int. J. Oncol. 38 (1) (2011) 97-103.

[41] C. Schichor, et al., Vascular endothelial growth factor A contributes to gliomainduced migration of human marrow stromal cells (hMSC), Exp. Neurol. 199 (2) (2006) 301-310.

[42] S. Vogel, et al., Migration of mesenchymal stem cells towards glioblastoma 
cells depends on hepatocyte-growth factor and is enhanced by aminolaevulinic acid-mediated photodynamic treatment, Biochem. Biophys. Res. Commun. 431 (3) (2013) 428-432.

[43] N. Shinojima, et al., TGF-beta mediates homing of bone marrow-derived human mesenchymal stem cells to glioma stem cells, Canc. Res. 73 (7) (2013) 2333-2344.

[44] T. Birnbaum, et al., Malignant gliomas actively recruit bone marrow stromal cells by secreting angiogenic cytokines, Journal of neuro-oncology 83 (3) (2007) 241-247.

[45] I.A. Ho, et al., Matrix metalloproteinase 1 is necessary for the migration of human bone marrow-derived mesenchymal stem cells toward human glioma, Stem Cell. 27 (6) (2009) 1366-1375.

[46] R. Kim, et al., Alternative new mesenchymal stem cell source exerts tumor tropism through ALCAM and N-cadherin via regulation of microRNA-192 and -218, Mol. Cell. Biochem. 427 (1-2) (2017) 177-185.

[47] Y. Gao, et al., p27 modulates tropism of mesenchymal stem cells toward brain tumors, Experimental and therapeutic medicine 1 (4) (2010) 695-699.

[48] J.G. Thomas, et al., Ionizing radiation augments glioma tropism of mesenchymal stem cells, J. Neurosurg. (2017) 1-9.

[49] D.W. Stuckey, K. Shah, Stem cell-based therapies for cancer treatment: separating hope from hype, Nat. Rev. Canc. 14 (10) (2014) 683-691.

[50] C.H. Ryu, et al., Gene therapy of intracranial glioma using interleukin 12 secreting human umbilical cord blood-derived mesenchymal stem cells, Hum. Gene Ther. 22 (6) (2011) 733-743.

[51] G. Xu, et al., Adenoviral-mediated interleukin-18 expression in mesenchymal stem cells effectively suppresses the growth of glioma in rats, Cell Biology International 33 (4) (2009) 466-474.

[52] J.H. Park, C.H. Ryu, M.J. Kim, S.S. Jeun, Combination therapy for gliomas using temozolomide and interferon-beta secreting human bone marrow derived mesenchymal stem cells, Journal of Korean Neurosurgical Society 57 (5) (2015) 323-328.

[53] C. Altaner, et al., Complete regression of glioblastoma by mesenchymal stem cells mediated prodrug gene therapy simulating clinical therapeutic scenario, J. Int. Cancer 134 (6) (2014) 1458-1465.

[54] C.H. Ryu, et al., Valproic acid enhances anti-tumor effect of mesenchymal stem cell mediated HSV-TK gene therapy in intracranial glioma, Biochem. Biophys. Res. Commun. 421 (3) (2012) 585-590.

[55] M. Roger, et al., Mesenchymal stem cells as cellular vehicles for delivery of nanoparticles to brain tumors, Biomaterials 31 (32) (2010) 8393-8401.

[56] S.M. Kim, et al., Potential application of temozolomide in mesenchymal stem cell-based TRAIL gene therapy against malignant glioma, Stem cells translational medicine 3 (2) (2014) 172-182.

[57] F. Djouad, et al., Immunosuppressive effect of mesenchymal stem cells favors tumor growth in allogeneic animals, Blood 102 (10) (2003) 3837-3844.

[58] S.G. Kang, et al., Cytotoxicity of rat marrow stromal cells against malignant glioma cells, Child's Nerv. Syst. : ChNS : official journal of the International Society for Pediatric Neurosurgery 21 (7) (2005) 528-538.

[59] S.G. Kang, et al., Cytotoxicity of human umbilical cord blood-derived mesenchymal stem cells against human malignant glioma cells, Child's Nerv. Syst. : ChNS : official journal of the International Society for Pediatric Neurosurgery 24 (3) (2008) 293-302.

[60] V.R. Dasari, et al., Upregulation of PTEN in glioma cells by cord blood mesenchymal stem cells inhibits migration via downregulation of the PI3K/ Akt pathway, PLoS One 5 (4) (2010) e10350.

[61] I.A. Ho, et al., Human bone marrow-derived mesenchymal stem cells suppress human glioma growth through inhibition of angiogenesis, Stem Cell. 31 (1) (2013) 146-155.

[62] S. Pacioni, et al., Human mesenchymal stromal cells inhibit tumor growth in orthotopic glioblastoma xenografts, Stem Cell Research \& Therapy 8 (1) (2017) 53.

[63] D. Bexell, et al., Bone marrow multipotent mesenchymal stroma cells act as pericyte-like migratory vehicles in experimental gliomas, Mol. Ther. : The Journal of the American Society of Gene Therapy 17 (1) (2009) 183-190.

[64] M. Roger, et al., In vitro and in vivo interactions between glioma and marrowisolated adult multilineage inducible (MIAMI) cells, Brain Research 1473 (2012) 193-203.

[65] B. Breznik, H. Motaln, M. Vittori, A. Rotter, T. Lah Turnsek, Mesenchymal stem cells differentially affect the invasion of distinct glioblastoma cell lines, Oncotarget 8 (15) (2017) 25482-25499.

[66] A.O. Pires, et al., Unveiling the differences of secretome of human bone marrow mesenchymal stem cells, adipose tissue-derived stem cells, and human umbilical cord perivascular cells: a proteomic analysis, Stem Cells and Development 25 (14) (2016) 1073-1083.
[67] K. Akimoto, et al., Umbilical cord blood-derived mesenchymal stem cells inhibit, but adipose tissue-derived mesenchymal stem cells promote, glioblastoma multiforme proliferation, Stem Cells and Development 22 (9) (2013) 1370-1386.

[68] W. Roth, et al., Locoregional Apo2L/TRAIL eradicates intracranial human malignant glioma xenografts in athymic mice in the absence of neurotoxicity, Biochem. Biophys. Res. Commun. 265 (2) (1999) 479-483.

[69] J. Lee, M. Hampl, P. Albert, H.A. Fine, Antitumor activity and prolonged expression from a TRAIL-expressing adenoviral vector, Neoplasia 4 (4) (2002) $312-323$.

[70] J. Liu, Y. Zhang, L. Bai, X. Cui, J. Zhu, Rat bone marrow mesenchymal stem cells undergo malignant transformation via indirect co-cultured with tumour cells, Cell Biochemistry and Function 30 (8) (2012) 650-656.

[71] X. Cui, J. Liu, L. Bai, J. Tian, J. Zhu, Interleukin-6 induces malignant transformation of rat mesenchymal stem cells in association with enhanced signaling of signal transducer and activator of transcription 3, Canc. Sci. 105 (1) (2014) 64-71.

[72] S. Ma, et al., Human umbilical cord mesenchymal stem cells inhibit C6 glioma growth via secretion of dickkopf-1 (DKK1), Mol. Cell. Biochem. 385 (1-2) (2014) 277-286.

[73] C. Yang, et al., Conditioned media from human adipose tissue-derived mesenchymal stem cells and umbilical cord-derived mesenchymal stem cells efficiently induced the apoptosis and differentiation in human glioma cell lines in vitro, BioMed Research International 2014 (2014), 109389.

[74] K. Kolosa, H. Motaln, C. Herold-Mende, M. Korsic, T.T. Lah, Paracrine effects of mesenchymal stem cells induce senescence and differentiation of glioblastoma stem-like cells, Cell Transplantation 24 (4) (2015) 631-644.

[75] A. Del Fattore, et al., Differential effects of extracellular vesicles secreted by mesenchymal stem cells from different sources on glioblastoma cells, Expet Opin. Biol. Ther. 15 (4) (2015) 495-504.

[76] H.Y. Lee, I.S. Hong, Double-edged sword of mesenchymal stem cells: cancerpromoting versus therapeutic potential, Canc. Sci. 108 (10) (2017) 1939-1946.

[77] X. Zhang, et al., Mesenchymal stem cell-derived extracellular vesicles: roles in tumor growth, progression, and drug resistance, Stem Cells International 2017 (2017), 1758139.

[78] I.A. Chistyakova, G.G. Poljanskaya, The influence of human fetal mesenchymal stem cells on glioma cell proliferation. The consequence of cellular crosstalk, Cell and Tissue Biology 9 (2) (2014) 71-78.

[79] A. Bajetto, et al., Different effects of human umbilical cord mesenchymal stem cells on glioblastoma stem cells by direct cell interaction or via released soluble factors, Front. Cell. Neurosci. 11 (2017) 312.

[80] I.C. Iser, et al., Conditioned medium from adipose-derived stem cells (ADSCs) promotes epithelial-to-mesenchymal-like transition (EMT-Like) in glioma cells in vitro, Mol. Neurobiol. 53 (10) (2016) 7184-7199.

[81] G.R. Onzi, et al., Analysis of the safety of mesenchymal stromal cells secretome for glioblastoma treatment, Cytotherapy 18 (7) (2016) 828-837.

[82] J. Vieira de Castro, et al., Impact of mesenchymal stem cells' secretome on glioblastoma pathophysiology, J. Transl. Med. 15 (1) (2017) 200.

[83] F.G. Teixeira, M.M. Carvalho, N. Sousa, A.J. Salgado, Mesenchymal Stem Cells Secretome: a New Paradigm for central Nervous System Regeneration? Cellular and Molecular Life Sciences, CMLS, 2013.

[84] C.A. Ribeiro, et al., The secretome of stem cells isolated from the adipose tissue and Wharton jelly acts differently on central nervous system derived cell populations, Stem Cell Research \& Therapy 3 (3) (2012) 18.

[85] P.C. Chagastelles, N.B. Nardi, M. Camassola, Biology and applications of mesenchymal stem cells, Sci. Prog. 93 (Pt 2) (2010) 113-127.

[86] A.J. Salgado, et al., Mesenchymal stem cells secretome as a modulator of the neurogenic niche: basic insights and therapeutic opportunities, Front. Cell. Neurosci. 9 (2015) 249.

[87] E. Favaro, et al., Human mesenchymal stem cell-derived microvesicles modulate $\mathrm{T}$ cell response to islet antigen glutamic acid decarboxylase in patients with type 1 diabetes, Diabetologia 57 (8) (2014) 1664-1673.

[88] A.M. Mikheev, et al., Periostin is a novel therapeutic target that predicts and regulates glioma malignancy, Neuro Oncol. 17 (3) (2015) 372-382.

[89] C.A. Formolo, et al., Secretome signature of invasive glioblastoma multiforme J. Proteome Res. 10 (7) (2011) 3149-3159.

[90] J. Yang, et al., SPOCK1 promotes the proliferation, migration and invasion of glioma cells through PI3K/AKT and Wnt/beta-catenin signaling pathways, Oncol. Rep. 35 (6) (2016) 3566-3576.

91] H.L. Goel, et al., GLI1 regulates a novel neuropilin-2/alpha6beta1 integrin based autocrine pathway that contributes to breast cancer initiation, EMBO Molecular Medicine 5 (4) (2013) 488-508. 